\title{
Efecto Antimicrobiano de Tres Cementos Selladores Endodónticos frente a Streptococcus mutans, Enterococcus faecalis y Candida albicans
}

\author{
Antimicrobial Effect of Three Sealant Endodontic Cements for \\ Streptococcus mutans, Enterococcus faecalis and Candida albicans
}

\author{
Katya Chambilla Torres $^{1} \&$ Marco Antonio Sánchez-Tito ${ }^{2}$
}

CHAMBILLA, T. K. \& SÁNCHEZ-TITO, M. A. Efecto antimicrobiano de tres cementos selladores endodónticos frente a Streptococcus mutans, Enterococcus faecalis y Candida albicans. Int. J. Odontostomat., 15(3):610-615, 2021.

RESUMEN: El objetivo del estudio fue evaluar el efecto antimicrobiano de tres cementos selladores endodónticos frente a Streptococcus mutans, Enterococcus faecalis y Candida albicans. Se usó el método de difusión en agar, realizando pozos de $5 \mathrm{~mm}$ de diámetro por $4 \mathrm{~mm}$ de profundidad, en donde se colocaron los diferentes cementos que fueron preparados según las indicaciones del fabricante. Para S. mutans y E. faecalis se empleó agar cerebro-corazón, mientras que para C. albicans se empleó agar tripticasa soya como medios de cultivo. Las placas fueron incubadas por 48 horas a $37^{\circ} \mathrm{C}$. Los halos de inhibición de crecimiento microbiano se midieron con un compás Vernier. Los resultados mostraron diferencias significativas entre todos los grupos $p<0,05$. El cemento a base de óxido de zinc y eugenol (Endofill) mostró el mejor efecto antimicrobiano contra las tres cepas con halos de inhibición de 23,283 $\pm 5,497 \mathrm{~mm}$ para S. mutans, 8,468 $\pm 0,755 \mathrm{~mm}$ para E. faecalis y $21,965 \pm 1,187 \mathrm{~mm}$ para C. albicans. Mientras que el cemento a base de resina (Vioseal) tuvo una actividad similar a Endofill solo para S. mutans $(21,262 \pm 2,602 \mathrm{~mm})(p>0,05)$, siendo bastante inferior para E. faecalis $(6,642 \pm$ $0,659 \mathrm{~mm})$ y $C$. albicans $(8,781 \pm 0,735 \mathrm{~mm})$. El cemento a base de hidróxido de calcio (Sealer 26) fue más efectivo frente a S. mutans $(13,010 \pm 2,006 \mathrm{~mm})$ que ante E. faecalis $(6,165 \pm 0,978 \mathrm{~mm})$ y C. albicans $(5,640 \pm 0,280 \mathrm{~mm})$. Como conclusión, el cemento a base de óxido de zinc y eugenol (Endofill) mostró el mejor efecto antimicrobiano contra las tres cepas, seguido de los cementos a base de resina (Vioseal) y finalmente hidróxido de calcio (Sealer 26).

PALABRAS CLAVE: Streptococccus mutans, Enterococcus faecalis, Candida albicans, cementos dentales.

\section{INTRODUCCIÓN}

Se estima que el fracaso del tratamiento endodóntico puede alcanzar entre el 14 al $18 \%$ de los casos y puede ocurrir inclusive desde los seis meses post tratamiento y hasta luego de 10 años (Solanki et al., 2019). Su ocurrencia está asociada a una variedad de factores como la persistencia de bacterias (intracanal y extracanal), llenado inadecuado del canal, sobre extensión en la obturación, sellado coronal inadecuado, canales accesorios no tratados, diseño deficiente de la cavidad de acceso y complicaciones en la instrumentación (escalones, perforaciones o instrumentos separados) (Toledo et al., 2016; Tabassum \& Khan, 2016; Yamaguchi et al., 2018).
La persistencia de microorganismos patógenos dentro del conducto radicular se debe a su capacidad de adherirse a la pared del conducto y al efecto de sus productos metabólicos. Estas características permiten que las bacterias colonicen áreas del conducto radicular, como los istmos, los túbulos dentinarios y las ramificaciones, consecuentemente dificultando su eliminación (Tabassum \& Khan, Lacerda et al., 2016). Los principales agentes patógenos asociados al fracaso del tratamiento endodóntico son Enterococcus faecalis (E. faecalis) y Streptococcus mutans (S. mutans), pudiendo encontrarse también la presencia de Candida albicans (C. albicans) (Del Fabbro et al., 2014).

\footnotetext{
${ }^{1}$ Cirujano Dentista, Práctica privada.

${ }^{2}$ Magister en Investigación Científica e Innovación, Escuela Profesional de Odontología, Facultad de Ciencias de la Salud, Universidad Privada de Tacna, Perú.
} 
La presencia de $S$. mutans en los conductos radiculares se relaciona con filtraciones en las restauraciones ya sean provisionales o permanentes. Esta bacteria al ser anaeróbica facultativa puede sobrevivir con o sin oxígeno, además tolera un pH bajo, asegurando su supervivencia dentro de los conductos radiculares (Zhang et al., 2012). Por otra parte, E. faecalis, puede sobrevivir durante un largo período sin el apoyo de otras bacterias a la escasez de nutrientes, invadiendo los túbulos dentinarios y adhiriéndose a la dentina. Además, altera las defensas del huésped, inhibe la acción de los linfocitos, posee enzimas líticas, citolisina, hialuronidasa y ácido lipoteicoico. Posee un inhibidor de la pompa de protones en funcionamiento (CCCP), permitiendo así regular el pH de esta bacteria frente a agentes desinfectantes (Prada et al., 2016). C. albicans tiene el potencial de cambiar el estado hidrofílico de su superficie celular al hidrofóbico; y además poseer proteinasas ácidas en la capa más externa de su pared celular, aumentando su adhesión a la superficie dentaria; su supervivencia post tratamiento de endodoncia se debe a que este hongo invade los túbulos dentinarios y es dentinofílico, sobreviviendo a los métodos de desinfección intraconducto (BernalTreviño et al., 2018).

En el área de endodoncia, una de las características más requeridas en los cementos selladores es la acción antimicrobiana, ya que se considera positiva y beneficiosa para la reducción del número de microorganismos patógenos presentes, eliminando la infección existente y evitando una reactivación microbiana futura (Prada et al.). Los cementos resinosos adquieren su acción antimicrobiana por la liberación de formaldehido dada durante la reacción de polimerización o por la liberación de componentes amina y resina epoxi, inhibiendo la síntesis citoplasmática (Asawaworarit et al., 2016). Los cementos selladores a base de hidróxido de calcio presentan actividad antimicrobiana debido la separación de los iones hidroxilo y calcio, alterando el gradiente de pH de la membrana citoplásmica (Pérez et al., 2014). Mientras que los cementos a base de óxido de zinc y eugenol lo obtienen por los grupos hidroxilos libres del eugenol, que alteran la permeabilidad de la membrana celular microbiana (Torres et al., 2019). Dalmia et al. (2018) encontraron que el cemento a base de resina posee mayor efecto antimicrobiano frente a $E$. faecalis, presentando un halo de inhibición de entre $19 \mathrm{~mm}$, en comparación al cemento a base de óxido de zinc y eugenol con un halo de $11 \mathrm{~mm}$. HerediaVeloz et al. (2017), por el contrario, encontró que el cemento a base de óxido de zinc y eugenol fue el de mayor efecto antimicrobiano frente a $E$. faecalis con un halo de $8,4 \mathrm{~mm}$, seguido por los cementos a base de resina con un halo de $7,7 \mathrm{~mm}$ y de hidróxido de calcio con $6,0 \mathrm{~mm}$.

Con base en los hallazgos previos, el objetivo del estudio fue comparar la actividad antimicrobiana frente a $S$. mutans, E. faecalis y $C$. albicans de tres cementos selladores endodónticos a base de resina, hidróxido de calcio y óxido de zinc y eugenol.

\section{MATERIAL Y MÉTODO}

Se realizó un estudio de tipo experimental in vitro, de corte transversal y analítico. Se obtuvo la autorización del Comité de Ética de la Facultad de Ciencias de la Salud de la Universidad Privada de Tacna bajo el registro: 038-FACSA/UPT. El cálculo de las repeticiones se realizó utilizando la ecuación de medias basada en la potencia ( $($ ) y confianza (a): siendo la confianza al $95 \%(1,96)$, la potencia de la prueba en un $80 \%(0,84)$ y la variación relativa de los halos 1 $\mathrm{mm}$. Se utilizaron 8 repeticiones por cada cemento.

Activación e inoculación de las cepas. Las cepas deS. mutans (ATCC ${ }^{\circledR} 25175^{\mathrm{TM}}$ ) y E. faecalis (ATCC $\AA$ $29212^{\mathrm{TM}}$ ) fueron reactivadas en una infusión nutritiva de caldo cerebro-corazón (BHI) e incubados por $24 \mathrm{~h}$ en aerobiosis a $37^{\circ} \mathrm{C}$. Los tubos se ajustaron a una escala 0,5 de McFarland lo que equivale a 1,5×108 UFC/mL. Se transfirieron $100 \mathrm{~mL}$ de cada suspensión microbiana en las placas Petri con agar cerebro-corazón (BHA). C. albicans fue cultivada en un caldo de tripticasa soya (TSB) a $37^{\circ} \mathrm{C}$ por $24 \mathrm{~h}$, para luego ser sembrada en placas Petri con agar tripticasa soya (TSA). Todos los medios de cultivos se obtuvieron de Liofilchem ${ }^{\circledR}$ (Roseto degli Abruzzi, TE, Italia).

Ensayo de difusión en pozo. Sobre el agar contenido en las placas Petri se realizaron 3 pozos de $5 \mathrm{~mm}$ de diámetro y $4 \mathrm{~mm}$ de profundidad con el uso de sacabocados estéril. Cada pozo fue rellenado con el material correspondiente, se emplearon los cementos endodónticos a base de resina (Vioseal ${ }^{\circledR}$ - Spident, Seúl, Corea), de hidróxido de calcio (Sealer 26, Dentsply Sirona, Industria y comercio Ltda. SP, Brasil) y óxido de zinc y eugenol (Endofill, Dentsply Sirona, Industria y comercio Ltda. SP, Brasil). Los cementos fueron prepararon de acuerdo a las recomendaciones de sus fabricantes. Enseguida, las placas Petri fueron incubadas a $37^{\circ} \mathrm{C}$ por $48 \mathrm{~h}$ en condiciones de 
aerobiosis. Los halos de inhibición formados sobre las placas Petri se midieron con un compás Vernier (Ubermann®).

Análisis de datos. El análisis estadístico fue procesado utilizando el software estadístico SPSS para Windows en su versión 25.0 (SPSS Inc., Chicago IL, EE.UU.). Se usaron las pruebas de Shapiro Wilk para constatar la normalidad de los datos y la prueba del análisis de la varianza (ANOVA) seguida de la prueba post hoc de Tukey para comparación de los datos. Se estableció un nivel de significancia del $5 \%$ para todas las pruebas.

\section{RESULTADOS}

La prueba ANOVA mostró que existieron diferencias significativas entre los halos de inhibición generados por los cementos y las cepas empleadas $(p<$ $0,05)$. En la Tabla I se muestra el promedio de las medidas de los halos de inhibición producidos por los cementos selladores. Endofill presentó mayor actividad antibacteriana frente a $S$. mutans $(23,283 \pm 6,642$ $\mathrm{mm})$ que Vioseal, aunque no existieron diferencias estadísticamente significativas $(p>0,05)$, mientras que los valores para Sealer 26 fueron estadísticamente inferiores $(p<0,05)$. Frente a $E$. faecalis, Endofill presentó mayor actividad antibacteriana $(8,468 \pm 0,755$ $\mathrm{mm}$ ) en comparación a los otros dos cementos $(p<0,05)$. En el caso de $C$. albicans, Endofill presentó actividad antifúngica muy superior $(21,965 \pm 1,187 \mathrm{~mm})$ que Sealer 26 y Vioseal $(p<0,05)$.

En las Figuras 1, 2 y 3 se observaron las comparaciones de los halos de inhibición generados por los cementos para cada una de las cepas estudiadas. En el caso de $S$. mutans, los halos de inhibición generados por Endofill y Vioseal fueron similares $(p>0,05)$ pero estadísticamente diferentes frente a Sealer 26 ( $p$ $<0,05)$. Para E. faecalis, los halos generados por
Endofill fueron superiores que los registrados para Vioseal y Sealer $26(p<0,05)$. Para $C$. albicans, los halos de inhibición generados por los tres cementos

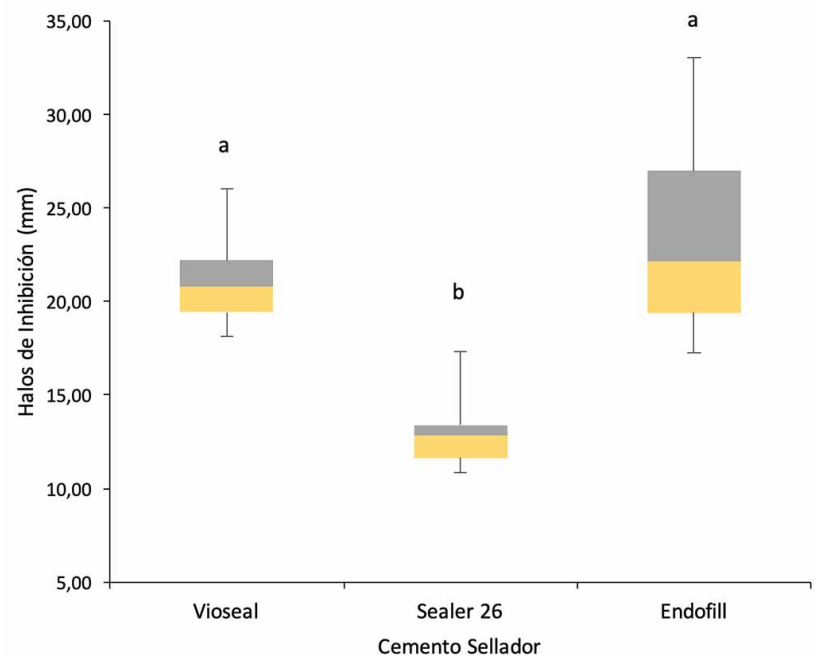

Fig. 1. Boxplot para la comparación de los halos de inhibición generados por los cementos selladores endodónticos frente a Streptococcus mutans. Letras distintas expresan diferencias significativas entre los halos de inhibición.

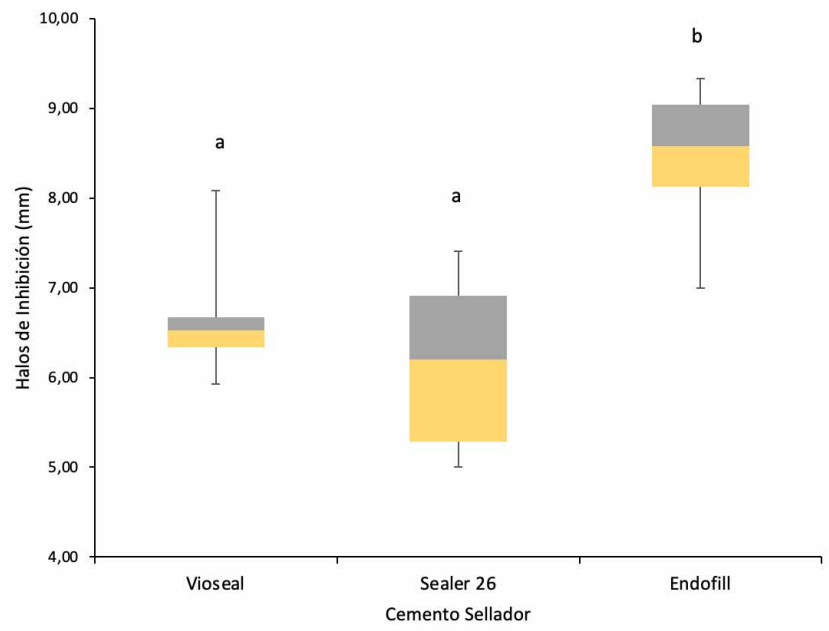

rig. L. Boxpiot para Ia comparacion de Ios nalos de InnıDIción generados por los cementos selladores endodónticos frente a Enterococcus faecalis. Letras distintas expresan diferencias significativas entre los halos de inhibición.

Tabla I. Promedio de halos de inhibición $(\mathrm{mm})$ de los cementos selladores endodónticos frente a Streptococcus mutans, Enterococcus faecalis, y Candida albicans.

\begin{tabular}{|c|c|c|c|c|c|c|c|}
\hline \multirow{3}{*}{ Cemento sellador } & \multicolumn{6}{|c|}{ Microorganismo } & \multirow{3}{*}{$\begin{array}{r}\text { Valor } p \\
0,000\end{array}$} \\
\hline & \multicolumn{2}{|c|}{ Streptococcus mutans } & \multicolumn{2}{|c|}{ Enterococcus faecalis } & \multirow{2}{*}{$\begin{array}{c}\text { Candida } \\
\text { Media. }\end{array}$} & \multirow{2}{*}{$\begin{array}{c}\text { albicans } \\
\text { D.E }\end{array}$} & \\
\hline & Media & D.E. & Media & D.E. & & & \\
\hline Vio & $21,262 a$ & 2,602 & $6,642^{\mathrm{a}}$ & 0,659 & $8,781^{\mathrm{a}}$ & 0,735 & \\
\hline Sealer 26 & $13,010 \mathrm{~b}$ & 2,006 & $6,165^{\mathrm{a}}$ & 0,978 & $5,640^{\mathrm{b}}$ & 0,280 & \\
\hline Endofill & $23,283 a$ & 5,497 & $8,468^{\mathrm{D}}$ & 0,755 & $21,965^{c}$ & 1,187 & \\
\hline
\end{tabular}

D.E.: desviación estándar. Letras distintas (en superíndice) expresan diferencias significativas entre los halos de inhibición generados por los cementos para cada cepa. 
selladores fueron estadísticamente diferentes $(p<$ 0,05). En la Figura 4 se muestran los halos de inhibición generados por los cementos para cada una de las cepas.

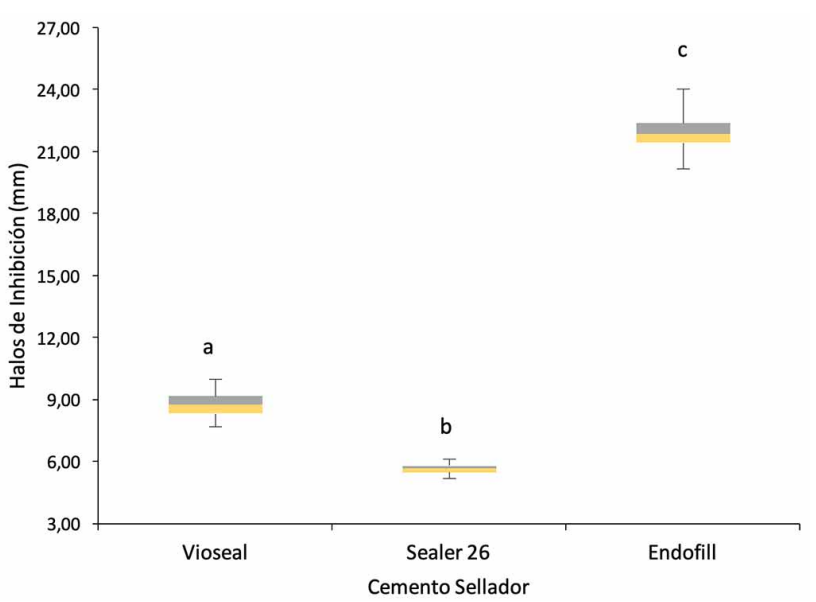

Fig. 3. Boxplot para la comparación de los halos de inhibición generados por los cementos selladores endodónticos frente a Candida albicans. Letras distintas expresan diferencias significativas entre los halos de inhibición.

\section{DISCUSIÓN}

A lo largo de los años, se han usado una diversa gama de cementos selladores de los sistemas de conductos radiculares. Estos cementos deben presentar propiedades relevantes como biocompatibilidad, radiopacidad, sellado hermético y una actividad antimicrobiana aceptable. Los cementos evaluados en este estudio fueron: Vioseal (a base de resina), Sealer 26 (a base de hidróxido de calcio) y Endofill (a base de óxido de zinc y eugenol).
En la última década diversos investigadores han estudiado las propiedades antimicrobianas de cementos selladores contra diversas cepas microbianas buscando demostrar la efectividad de esta propiedad. Farmakis et al. (2012), encontraron que un cemento a base de resina $(\mathrm{AH}-26)$ generó halos de inhibición contra $E$. faecalis de $19 \mathrm{~mm}$ en promedio, mayores a los generados por un cemento a base de $\mathrm{ZnO}+$ Eugeno I (Roth 601) y otros cementos a base de resina (Topseal, AH-plus y EndoREZ); y contra Proteus vulgaris fue el cemento a base de $\mathrm{ZnO}+$ Eugenol (Roth 601) el que obtuvo mejor resultados $(24 \mathrm{~mm}$ ) seguido por los cementos a base de resina (AH-26, Topseal, AH-plus y EndoREZ).

Dalmia et al. encontraron que el cemento a base de $\mathrm{Ca}(\mathrm{OH}) 2$ (Sealapex) posee una mejor actividad antibacteriana frente a $E$. faecalis $(13,33$ a $14,66 \mathrm{~mm}$ ) seguido por el cemento a base de resina (AH Plus) y el de $\mathrm{ZnO}+$ Eugenol (Tubliseal). Por otro lado, Heredia-Veloz et al. encontraron que el cemento a base de $\mathrm{ZnO}+$ Eugenol (Grossfar) fue el de mayor actividad antibacteriana frente a la misma cepa $(8,4 \mathrm{~mm})$, seguido por el cemento a base de resina (Topseal) y el de $\mathrm{Ca}(\mathrm{OH}) 2$ (Sealapex), que a pesar de ser un cemento de la misma marca comercial que en el estudio de Dalmia et al., no obtuvo resultados favorables. En el presente estudio se obtuvieron resultados similares a los de Heredia-Veloz et al., observando que el cemento a base de $\mathrm{ZnO}+$ Eugenol (Endofill) tuvo mejor actividad antimicrobiana contra $E$. faecalis, seguido por los cementos a base de resina (Vioseal) y a base de $\mathrm{Ca}(\mathrm{OH}) 2$ (Sealer 26 ).

En la investigación de Bhargava et al. (2015), encontraron que el cemento a base de $\mathrm{ZnO}+$ Eugenol (EndoflasFS) tuvo mayor actividad antimicrobiana contra $E$. faecalis $(11,90 \mathrm{~mm})$ como para $C$. albicans $(10,12 \mathrm{~mm})$, seguido por el cemento a base de $\mathrm{Ca}(\mathrm{OH}) 2$ (Apexit Plus) y el de resina

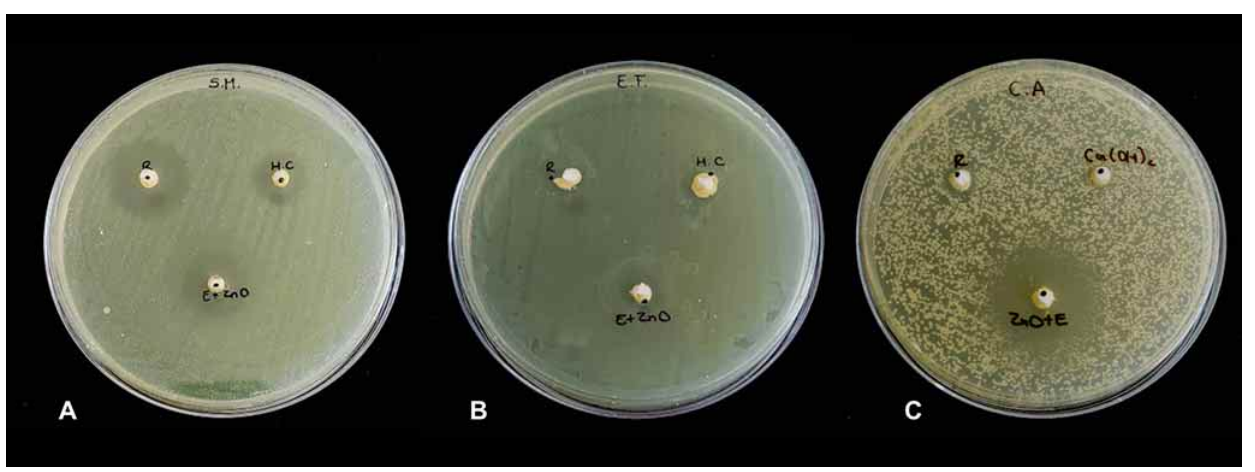

Fig. 4. Halos de inhibición de crecimiento microbiano generados por los cementos selladores endodónticos. A. frente a S. mutans. B. frente a E. faecalis. C. frente a C. albicans. 
(EndoREZ) con medidas muy inferiores. Monajemzadeh et al. (2017), encontraron que el cemento a base de resina (AH 26) fue el de mejor actividad antimicrobiana contra C. albicans $(26 \mathrm{~mm})$ mientras que el de $\mathrm{ZnO}+$ Eugenolno fue capaz de inhibir su crecimiento; en el caso de $S$. mutans obtuvo resultados iguales entre ambos cementos (20 $\mathrm{mm}$ ). En nuestro estudio se observaron resultados similares a los de Bhargava et al. contra C. albicans, siendo el cemento a base de $\mathrm{ZnO}+$ Eugenol (Endofill) en cemento con mayor poder de inhibición del crecimiento fúngico $(21,965 \mathrm{~mm})$.

El cemento sellador a base de resina (Vioseal), posee una actividad antimicrobiana debido a la liberación de componentes no polimerizados como la amina y resina epoxi (epóxido) inhibiendo así la síntesis nuclear microbiana (división celular) y la del metabolito metionina, a su vez esencial para la síntesis citoplasmática. Este cemento no libera formaldehído en su proceso de polimerización, mejorando así su biocompatibilidad, pero disminuyendo relativamente su actividad antimicrobiana. (Asawaworarit et al.; Ramachandra et al., 2016). El cemento sellador a base de hidróxido de calcio (Sealer 26), obtiene su actividad antimicrobiana debido a la separación de los iones hidroxilo y calcio, alterando el gradiente de $\mathrm{pH}$ de la membrana citoplásmica microbiana, produciendo efectos como la desnaturalización de las proteínas, (por la elevada concentración de iones hidroxilo sobre las proteínas), biosíntesis de lípidos, daño del ADN y de la membrana citoplasmática microbiana (Pérez et al.; Mohammadi et al., 2011). El cemento sellador a base de óxido de zinc y eugenol (Endofill) es un compuesto lipofílico y presenta grupos hidroxilos libres del eugenol que dañan la membrana celular microbiana al alterar su permeabilidad seguida de la fuga del contenido celular; además de la inhibición de la actividad de las enzimas adenosina trifosfatasa (ATPasa) (Torres et al.; Dragland et al., 2019).

Los hallazgos de los estudios previos abren una variedad de posibilidades de investigaciones futuras; en las que se puedan evaluar la actividad antimicrobiana de diversas marcas de cementos frente a diversas cepas microbianas propias de los conductos radiculares generadoras de infecciones endodónticas; bajo distintas metodologías, con el fin de facilitar la elección de alguno de estos cementos por el clínico al momento de realizar un tratamiento endodóntico.

\section{CONCLUSIONES}

Como conclusión y bajo las limitaciones del presente estudio in vitro, podemos señalar que se encontraron diferencias entre el efecto antimicrobiano entre los tres cementos selladores usados en este estudio. El sellador a base de óxido de Zinc y eugenol (Endofill) mostró un efecto antimicrobiano superior contra las tres cepas, seguido del cemento a base de resina (Vioseal) y el menor efecto antimicrobiano correspondió al cemento a base de hidróxido de calcio (Sealer 26).

CHAMBILLA, T. K. \& SÁNCHEZ-TITO, M. A. Antimicrobial effect of three sealant endodontic cements for Streptococcus mutans, Enterococcus faecalis and Candida albicans. Int. J. Odontostomat., 15(3):610-615, 2021.

ABSTRACT: The objective of the study was to evaluate the antimicrobial effect of three endodontic sealant cements for Streptococcus mutans, Enterococcus faecalis and Candida albicans. The agar diffusion method was used, making wells $5 \mathrm{~mm}$ in diameter by $4 \mathrm{~mm}$ deep, where the different cements that were prepared according to the manufacturer's instructions were placed. For S. mutans and E. faecalis, brain-heart agar was used, while for $C$. albicans trypticase soy agar was used as culture media. The plates were incubated for 48 hours at $37^{\circ} \mathrm{C}$. The microbial growth inhibition halos were measured with a Vernier compass. The results showed significant differences between all groups $p<0.05$. The cement based on zinc oxide and eugenol (Endofill) showed the best antimicrobial effect against the three strains with inhibition halos of $23.283 \pm 5.497 \mathrm{~mm}$ for $S$. mutans, $8.468 \pm 0.755$ $\mathrm{mm}$ for $E$. faecalis and $21.965 \pm 1.187 \mathrm{~mm}$ for $C$. albicans. While the resin-based cement (Vioseal) had a similar activity to Endofill only for $S$. mutans $(21.262 \pm 2.602$ $\mathrm{mm})(p>0.05)$, being considerably lower for $E$. faecalis $(6.642 \pm 0.659 \mathrm{~mm})$ and C. albicans $(8.781 \pm 0.735 \mathrm{~mm})$. Calcium hydroxide-based cement (Sealer 26) was more effective against $S$. mutans $(13.010 \pm 2.006 \mathrm{~mm})$ than against $E$. faecalis $(6.165 \pm 0.978 \mathrm{~mm})$ and $C$. albicans $(5.640 \pm 0.280 \mathrm{~mm})$. In conclusion, cement based on zinc oxide and eugenol (Endofill) showed the best antimicrobial effect against the three strains, followed by resin-based cements (Vioseal) and finally calcium hydroxide (Sealer 26).

KEY WORDS: Streptococccus mutans, Enterococcus faecalis, Candida albicans, dental cements. 


\section{REFERENCIAS BIBLIOGRÁFICAS}

Asawaworarit, W.; Yachor, P.; Kijsamanmith, K. \& Vongsavan N. Comparison of the apical sealing ability of calcium silicate-based sealer and resin-based sealer using the fluid-filtration technique. Med. Princ. Pract., 25(6):561-5, 2016.

Bhargava, R.; Shashikala, K. \& Bhargava R. Antibacterial efficacy of three root canal sealers againts Enterococcus faecalis and Candida albicans: An in vitro study. SRM. J. Res. Sci., 6(3):1704, 2015.

Bernal-Treviño, A.; González-Amaro, A. M.; Méndez, G. V. \& Pozos-Guillen, A. Frecuencia de Candida en conductos radiculares de dientes con infección endodóntica primaria y persistente. Rev. Iberoam. Micol., 35(2):78-82, 2018.

Dalmia, S.; Gaikwad, A.; Samuel, R.; Aher, G.; Gulve, M. \& Kolhe, $S$. Antimicrobial efficacy of different endodontic sealers against Enterococcus faecalis: An in vitro study. J. Int. Soc. Prev. Community Dent., 8(2):104-9, 2018.

Del Fabbro, M.; Samaranayake, L. P.; Lolato, A.; Weinstein, T.; \& Taschieri, S. Analysis of the secondary endodontic lesions focusing on the extraradicular microorganisms: an overview. J. Investig. Clin. Dent., 5(4):245-54, 2014.

Dragland, I. S.; Wellendorf, H.; Kopperud, H.; Stenhagen, I. \& Valen $\mathrm{H}$. Investigation on the antimicrobial activity of chitosan-modified zinc oxide-eugenol cement. Biomater. Investig. Dent., 6(1):99106, 2019

Farmakis, E. T.; Kontakiotis, E. G.; Tseleni-Kotsovili, A. \& Tsatsas, V. G. Comparative in vitro antibacterial activity of six root canal sealers against Enterococcus faecalis and Proteus vulgaris. J. Investig. Clin. Dent., 3(4):271-5, 2012.

Heredia-Veloz, D.; Abad-Coronel, D. \& Villavicencio-Caparó, E. Eficacia antibacteriana de tres selladores endodónticos frente al Enterococcus faecalis. Rev. Estomatol. Herediana, 27(3):13240, 2017.

Lacerda, L. S. M. F.; Coutinho, M. T.; Barrocas, D.; Rodrigues, T. J.; Vidal., F. Infecção secundária e persistente e sua relação com o fracasso do tratamento endodôntico. Rev. Bras. Odontol., 73(3):212-7, 2016.

Mohammadi, Z. \& Dummer, P. M. Properties and applications of calcium hydroxide in endodontics and dental traumatology. Int. Endod. J., 44(8):697-730, 2011.

Monajemzadeh, A.; Ahmadi Asoor, S.; Aslani, S. \& Sadeghi-Nejad B. In vitro antimicrobial effect of different root canal sealers against oral pathogens. Curr. Med. Mycol., 3(2):7-12, 2017.

Pérez, M. A.; Guerrero, I. J. \& Celis, R. L. Efecto del eugenol residual en los conductos radiculares sobre la adhesión de endopostes lumínicos prefabricados, cementados con resina compuesta. Rev. Odont. Mex., 18(1):14-8, 2014.

Prada, I.; Micó-Muñoz, P.; Giner-Lluesma, T.; Micó-Martínez, P.; Collado-Castellano, N. \& Manzano-Saiz, A. Influence of microbiology on endodontic failure. Literature review. Med. Oral Patol. Oral Cir. Bucal, 24(3):264-372, 2016.

Ramachandra, P. K. M.; Krishnegowda, S. C.; Jaganath, B. M.; Rudranaik, S.; Manjula, C. G.; Kurup, N. B. \& Madanan, S. In vitro comparative evaluation of the antibacterial and antifungal activities of different root canal sealers against endodontic pathogens. Int. J. Prev. Clin. Dent. Res., 3(4):261-6, 2016.

Solanki, V.; Mnasuri, R.; Attur, K.; Vachhani, K., \& Kubavat, R. Endodontic treatment failure \& its management: A review. Natl. J. Integr. Res. Med., 10(3):68-74, 2019.

Tabassum, S. \& Khan, F. Failure of endodontic treatment: The usual suspects. Eur. J. Dent., 10(1):144-7, 2016.

Toledo, L.; Alfonso, M. \& Barreto, E. Evolución del tratamiento endodóntico y factores asociados al fracaso de la terapia. Medicentro Electrónica, 20(3):202-8, 2016.
Torres, F. F. E.; Guerreiro-Tanomaru, J. M.; Bosso-Martelo, R.; Espir, C. G.; Camilleri, J. \&Tanomaru-Filho, M. Solubility, porosity, dimensional and volumetric change of endodontic Sealers. Braz. Dent. J., 30(4):368-73, 2019.

Yamaguchi, M.; Noiri, Y.; Itoh, Y.; Komichi, S.; Yagi, K.; Uemura, R.; Naruse, H.; Matsui, S.; Kuriki, N.; Hayashi, M. \& Ebisu, S. Factors that cause endodontic failures in general practices in Japan. BMC Oral Health, 18(1):70, 2018.

Zhang, C.; Hou, B. X.; Zhao, H. Y.; \& Sun Z. Microbial diversity in failed endodontic root-filled teeth. Chin. Med. J., 125(6):1163-8, 2012.

Dirección para correspondencia:

Marco A. Sánchez-Tito

Facultad de Ciencias de la Salud

Universidad Privada de Tacna

Av. Jorge Basadre Grohmann s/n

Tacna

PERÚ

E-mail: marcosanchez2183@gmail.com 